\title{
Effect of Chest Compression Leaning on Accelerometry Waveforms
}

\author{
James K Russell, Dana Zive, Mohamud Daya \\ Oregon Health \& Science University, Portland OR, USA
}

\begin{abstract}
$C P R$ monitors provide feedback on rate, depth and release force (RF) of chest compressions. Excessive $R F$ ("leaning") impedes venous return, reducing blood flow. Available monitors detect leaning with a force sensor, an expensive component. Our objective was to determine whether leaning, like rate and depth, could be detected through the accelerometry signal alone.

Brief intervals of accelerometry signals centered on force minima were extracted from chest compressions recorded with CPR monitors used in 289 out-of-hospital cardiac arrest in the Portland metropolitan region from 2009 - 2015. Evidence for effects of leaning was sought with various neural networks. Testing was done with waveforms extracted from 147 additional cases.

A cascadeforward network with 2 hidden layers outperformed simpler alternatives.

Testing yielded $88.6 \%$ correct classifications. Cases with zero $R F$ were identified correctly as non-leaning in 99.9\% of 123714 cases.

Accelerometry in the vicinity of the release point provides information about the force at release and warrants further investigation.
\end{abstract}

\section{Introduction}

Cardiopulmonary resuscitation (CPR) contributes to survival from cardiac arrest, especially when initiated early, including by non-professional volunteer responders with limited training[1,2]. Key characteristics of highquality CPR include a rate between 100 to $120 \mathrm{cpm}$, depth of 2-2.4 inches and complete chest wall recoil[3]. CPR monitors provide feedback on rate, depth and release force (RF) of chest compressions. Excessive RF ("leaning") is common in practice and impedes chest wall recoil, venous return, and blood flow[4]. Leaning can be reduced with real-time feedback [5,6]. Available monitors that detect leaning include a force sensor, a relatively expensive component, for that purpose. Since rate and depth feedback can be provided using only an accelerometer, our objective was to determine whether leaning could also be detected from the accelerometry signal.

Development of a less expensive CPR feedback monitor that included leaning correction might help improve the quality of CPR with more widely available monitors that lack force sensors.

A relationship between accelerometry and leaning if any is unknown. We sought evidence for one using neural networks configured for pattern recognition. Theoretically, sufficiently large neural networks can be trained to recognize any existing pattern[7]. Excessively complex networks may be overtrained to a particular data set, and not generalize well. We surveyed a range of neural network architectures ("nnets") using data from CPR monitors used in out-of-hospital cardiac arrest treatment. These monitors included force sensors, which directly measure leaning, as well as accelerometers.

\section{Methods}

Brief intervals $(0.32 \mathrm{~s})$ of accelerometry signals centered on force minima (i.e. the point of release) were extracted from a training database of 487,077 compressions from a randomly selected subset $(n=289)$ of recordings from Philips MRx defibrillators equipped with CPR monitors used out-of-hospital in the Portland metropolitan region from 2009 - 2015. Accelerometry, recorded at 250 sps or 100 sps, was resampled as needed to $100 \mathrm{sps}$ for uniformity. Leaning was defined as present (release force $>=2.5 \mathrm{~kg})^{5}$, absent $(<1 \mathrm{~kg})$ or intermediate. Cases with evident recording anomalies were removed, leaving 486,789 compressions in the Training database.

We sought a classifier distinguishing leaning from non-leaning as a pattern recognition problem using the Matlab ${ }^{\circledR}$ Neural Networks Toolbox. Intermediate compressions were excluded during training. Testing was done with 251,117 additional waveforms extracted from 147 additional cases.

Two types of neural network architectures ("nnets") were examined: feedforward (FF) (each layer informed by only the immediately prior layer), and cascadeforward (CF), (each layer informed by all prior layers).

We surveyed performance of a range of FF nnets with up to 4 hidden layers, covering a binary exponential sequence of numbers of nodes in the $1^{\text {st }}$ layer $(2,4,8$, $16, \ldots, 512)$, and successively adding layers to the bestperforming instances of each previous design and its simpler neighbors (145 nnets). 


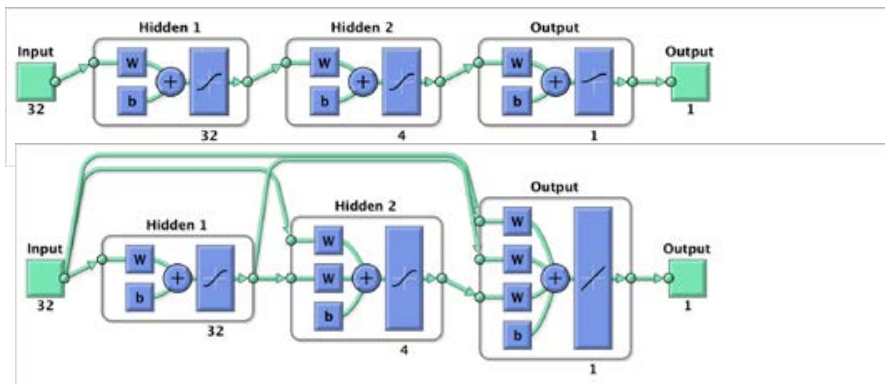

Figure 1. Feedforward (above) and cascadeforward (below) topologies with $321^{\text {st }}$ and $42^{\text {nd }}$ hidden layer nodes ([32 4]).

Interim results showed that fan-in architectures outperformed same-sized fan-out architectures and that flat architectures (same number of nodes in $1^{\text {st }}$ and $2^{\text {nd }}$ hidden layers) offered little if any advantage, so only fanin networks were explored further. We explored fan-in CF networks with 2 hidden layers and $1^{\text {st }}$ layers of up to 32 nodes (11 nnets). FF networks were implemented with the Matlab function "patternnet" trained with resilient backpropagation ("rp"), as recommended for patternnet. CF networks were implemented with the Matlab function cascadeforwardnet trained with Bayesian regularization backpropagation ("Brp"), as recommended for cascadeforwardnet. Selected FF nnets were also trained with Brp to examine the relative impacts of network structure and training method. Each nnet was trained 5 times independently. During each training session, all coefficients were re-initialized, $70 \%$ of the training cases were randomly assigned for learning, $15 \%$ for progress assessment and $15 \%$ for measurement of accuracy. Training proceeded until progress in performance stopped or 1000 epochs passed. Networks were evaluated on the basis of their median accuracy (\% correct classifications) and the stability of their accuracy over training sessions. Nnets with a range of accuracies over the 5 sessions of $>$ $1 \%$ were discarded as prone to overtraining (not generalizable). The most accurate examined CF network, having 32 nodes in the $1^{\text {st }}$ hidden layer, 16 in the $2^{\text {nd }}$ hidden layer ([32 16]) was assessed in more detail with the test database of 147 cases.

\section{Results}

Leaning is relatively uncommon (12\%) in the training dataset, reflecting high quality of responder training and realtime feedback. Avoidance of leaning is common (88\%). Most (61\%) compressions had RF $<1 \mathrm{~kg}$.

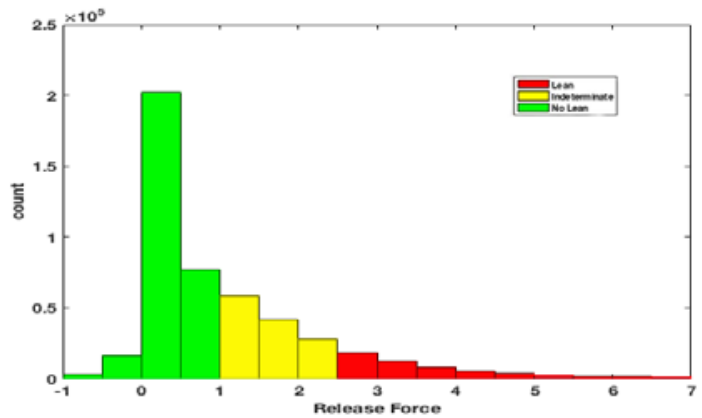

Figure 2. Observed release forces, Training database

The proportions of these types was essentially the same in the Test database. The difference between accelerometry signals with and without leaning is subtle and undescribed. No signal features are known or readily apparent, warranting use of pattern recognition analysis.
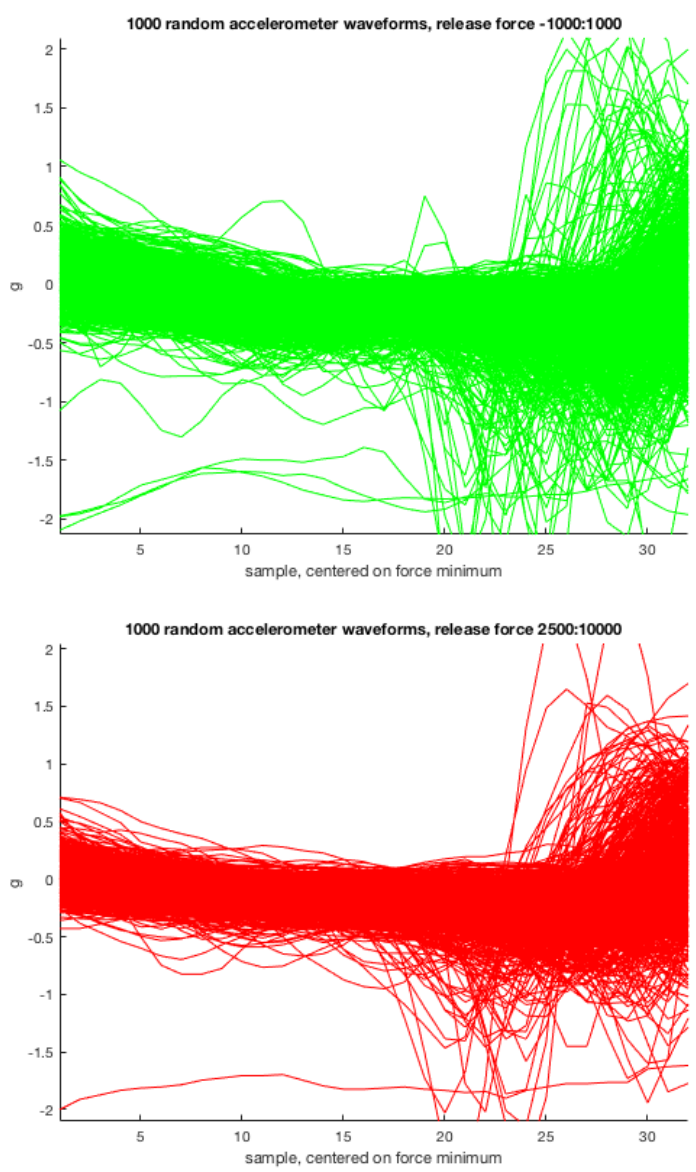

Figure 3. Accelerometry signals without (above), with leaning (below). $1 \mathrm{~g}$ removed for gravity. 
With the FF nnets trained with rp, within each group (1, 2, 3 or 4 hidden layers) median accuracy increased with network size up to a point, after which susceptibility to overtraining was evident. The optimal FF nnet was 64 nodes in a single hidden layer (86.8\% accuracy), a performance matched but not exceeded by some multiple hidden layer designs ([32 16 4], [32 16 8]).

Only the smallest ([ $\left.\left[\begin{array}{ll}4 & 2\end{array}\right]\right)$ CF networks showed susceptibility to overtraining. All generalizable CF nnets outperformed all generalizable FF nnets trained with rp. Selected FF nnets trained with Brp matched the performance of similarly structured CF nnets, suggesting that it is the regularization method during training more that the internal structure that most affects performance. CF performance increased steadily with size, reaching 88.6\% with the largest examined design ([32 16]). The [32 16] CF network with median performance used for further analysis.

The output layer of a neural network produces a continuous value transformed to lie between 0 and 1 . Conventionally, values $>=0.5$ are regarded as identified by the neural network, those $<0.5$ as rejected.

On this basis, and using the conventional limit of 2.5 $\mathrm{kg}$ for leaning, with the test database this network yielded overall sensitivity $=51 \%$, specificity $=91 \%$.

The correct interpretation of intermediate forces is unclear, and it may be useful to define leaning as neural net output $>=0.75$, no leaning as neural net output $<0.25$, and the remainder as indeterminate.

With this approach, only $0.6 \%$ of non-leaning cases are classified as leaning, though only $26.1 \%$ of leaning cases are classified as leaning. In fact, the neural net output is a continuous variable, and it tracks residual force generally smoothly:

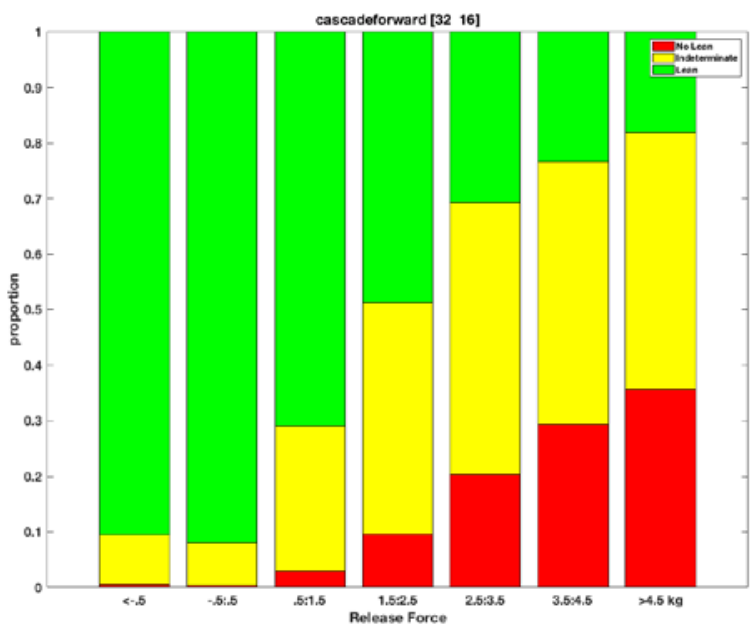

Figure 4. Leaning classification over the range of RF

Only $0.7 \%$ of the most common class, residual force between 0 and $0.5 \mathrm{~kg}$, are misclassified as leaning.
Specificity for the $25 \%$ of Test cases with RF $=0$ was 99.9\%.

\section{Discussion}

For adult patients, leaning is conventionally defined as $>$ $2.5 \mathrm{~kg}$ of residual force[4], but the impact of sternal force on hemodynamics is continuous, no safe limit has been documented, and optimal treatment of intermediate levels of residual force is unclear.

A CPR coaching application might seek to constrain negative feedback to the user about leaning to the minimum consistent with providing useful guidance, and to avoid misclassification of non-leaning compressions to reduce confusion and maintain user confidence. Serial analysis with hysteresis unaffected by indeterminate measures could possibly support effective coaching with moderate latency. At a nominal compression rate of 100 cpm 10 compressions are available in $6 \mathrm{~s}$.

The present analysis does not identify the optimal classifier for underlying such an application, but it does demonstrate that the accelerometry signal in the vicinity of the release point provides considerable information about the force at release. Further studies exploring alternative measures and classification algorithms based on this signal are warranted.

\section{References}

[1] Sasson C, Rogers MAM, Dahl J, Kellermann AL. Predictors of survival from out-of-hospital cardiac arrest: a systematic review and meta-analysis. Circulation: Cardiovascular Quality and Outcomes 2010;3;63-81.

[2] Iwami T, Kawamura T, Hiraide A, Berg RA, Hayashi Y, Nishiuchi T, Kajino K, Yonemoto N, Yukioka H, Sugimoto H, Kakuchi H, Sase K, Yokoyama H, Nonogi H. Effectiveness of bystander-initiated cardio-only resuscitation for patients with out-of-hospital cardiac arrest Circulation 2007;116;2900-7.

[3] Kleinman ME, Brennan EE, Goldberger ZD, Swor RA, Terry M, Bobrow BJ, Gazmuri RJ, Travers AH, Rea T. Part 5: Adult basic life support and cardiopulmonary resuscitation quality: 2015 American Heart Association Guidelines Update for Cardiopulmonary Resuscitation and Emergency Cardiovascular Care. Circulation 2015;132 Suppl 2, S414-35.

[4] Niles DE, Sutton RM, Nadkarni VM, Glatz A, Zuercher M, Maltese MR, Eilevstjonn J, Abella BS, Becker LB, Berg RA. Prevalence and hemodynamic effects of leaning during CPR. Resuscitation 2011;82 Suppl 2, S23-S26.

[5] Niles D, Nysaether J, Sutton R, Mishisaki A, Abella BS, Arbogast K, Maltese MR, Berg RA, Helfaer M, Nadkarni V. Leaning is common during in-hospital pediatric CPR, and decreased with automated corrective feedback. Resuscitation 2009;80;553-7.

[6] Buleon C, Delaunay J, Parienti J-J, Halbout L, Arrot X, Gerard J-L, Hanouz J-L. Impact of a feedback device on chest compression quality during extended manikin 
cardiopulmonary resuscitation: a randomized crossover study. The American Journal of Emergency Medicine 2016;34;1754-60.

[7] Hornik K, Stinchcombe M, White H. Multilayer feedforward networks are universal approximators. Neural Networks 1989;2;359-66.

Address for correspondence.

James K. Russell

Department of Emergency Medicine

Oregon Health Sciences University

3181 SW Sam Jackson Park Rd.

Portland OR 97239

james.k.russell@mac.com. 